\title{
Stability Analysis of Dual Solutions Magnetohydrodynamics Stagnation-Point Flow of Carreau Fluid Towards a Stretching/Shrinking Surface with Induced Magnetic Field and Convective Boundary Conditions
}

\author{
Mohamad Mustaqim Junoh ${ }^{1 *}$, Fadzilah Md Ali ${ }^{1,2}$, Norihan Md Ariffin ${ }^{1,2}$ and Norfifah Bachok ${ }^{1,2}$ \\ ${ }^{1}$ Institute for Mathematical Research, Universiti Putra Malaysia, 43400 UPM Serdang, Selangor, \\ Malaysia \\ ${ }^{2}$ Department of Mathematics, Faculty of Science, Universiti Putra Malaysia, 43400 UPM Serdang, \\ Selangor, Malaysia
}

\begin{abstract}
The problem of the steady two-dimensional magnetohydrodynamics (MHD) stagnation-point flow of Carreau fluid towards a permeable stretching/shrinking sheet is studied. The effect of the induced magnetic field and convective boundary conditions are taken into account. The nonlinear partial differential equations are transformed into nonlinear ordinary differential equations by using similarity transformations. The transformed governing equations are solved numerically via the boundary value problem solver (bvp4c) in MATLAB software. Numerical solutions for the physical quantities as well as the velocity and temperature profiles are obtained. It is found that dual solutions exist for a certain range of the controlling parameter. Therefore, a stability analysis is performed to determine which solution is linearly stable and valid physically.
\end{abstract}

Keywords: stagnation-point flow, Carreau fluid, MHD, dual solutions, stability analysis

\section{INTRODUCTION}

In view of far-flung applications, a lot of interest has been shown by the researchers towards the study of Carreau fluid. Carreau fluid is a type of generalized Newtonian fluid where viscosity depends upon the shear rate, the Carreau Viscosity Model (CVM) is useful in describing the flow behaviour of fluids in system at very low or high shear rate region. Initially invented by Pierre J. Carreau, his rheological model has garnered great attention nowadays (Carreau, 1972). Earlier, Khellaf and Lauriat (2000) investigated the heat transfer in non-Newtonian Carreau fluid between rotating concentric vertical cylinders and used SIMPLER algorithm to solve the resulting system.
Later, Khan and Hashim (2015) studied the Carreau viscosity model and then they use it to obtain a mathematical model for the Carreau fluid flow problem. They found that the increasing in values of stretching parameter thinning the boundary layer thickness while opposite effect occurs for the thermal boundary layer thickness. Malik et. al., (2014) examined the Carreau fluid flow through porous medium with pressure dependent viscosity. Hayat et. al., (2014) provided a homotopy analysis method to solve the problem of two-dimensional boundary layer flow of Carreau fluid pass a permeable stretching sheet to acquire the numerical result. In another study, Suneetha and Gangadhar (2015) discussed the effect of thermal radiation on a two-dimensional stagnation-point

*Corresponding author's e-mail: mohamadmustaqimjunoh@gmail.com 
flow of an incompressible magnetohydrodynamic Carreau fluid toward a shrinking surface in the presence of convective boundary condition. The study on the impact of magnetic field on stagnation point flow of Carreau fluid was done by Khan et al. (2016). Recently, Khan et. al., (2018) solved the problem of non-Newtonian Carreau fluid flow over a nonlinear inclined shrinking surface in the presence of infinite shear rate viscosity.

This present study is an extension work from Akbar et. al., (2014), where this study explores the presence of induced magnetic field on magnetohydrodynamics (MHD) stagnation point flow of Carreau fluid on shrinking/stretching sheet with the convective boundary conditions and performing a stability analysis of dual solutions since the solution is non-unique. Purpose of conducting a stability analysis is to determine which solution (first or second) is stable and physically realizable. Many works on stability analysis has been done by researchers such as Merkin (1985), Weidman et al. (2006), Adnan and Arifin (2017) and Junoh et. al., (2018).

\section{MATHEMATICAL FORMULATION}

Consider the steady two dimensional stagnation point flow of an incompressible Carreau fluid over a wall coinciding with plane $y=0$, the flow is being confined to $y>0$. The flow is generated due to the linear stretching. Extra stress tensor for Carreau fluid is,

$$
\bar{\tau}_{i j}=\eta_{0}\left[1+\frac{(n-1)}{2}(\Gamma \overline{\dot{\gamma}})^{2}\right] \overline{\dot{\gamma}}_{i j}
$$

in which $\bar{\tau}_{i j}$ is the extra stress tensor, $\eta_{0}$ is the zero shear rate viscosity, $\Gamma$ is the time constant, $n$ is the power law index and $\overline{\dot{\gamma}}$ is defined as

$$
\bar{\gamma}_{i j}=\sqrt{\frac{1}{2} \sum_{i} \sum_{j} \overline{\dot{\gamma}}_{i j} \overline{\dot{\gamma}}_{j i}}=\sqrt{\frac{1}{2} \prod} .
$$

Here $\prod$ is the second invariant strain tensor. Flow, induced magnetic and energy equations for Carreau fluid model after applying the boundary layer approximations can be defined as follows

$$
\begin{gathered}
\frac{\partial u}{\partial x}+\frac{\partial v}{\partial y}=0 \\
\frac{\partial H_{1}}{\partial x}+\frac{\partial H_{2}}{\partial y}=0
\end{gathered}
$$

$$
\begin{gathered}
u \frac{\partial u}{\partial x}+v \frac{\partial u}{\partial y}=v \frac{\partial^{2} u}{\partial y^{2}}+u_{e} \frac{d u_{e}}{d x}-\frac{\mu H_{e}}{4 \pi \rho} \frac{d H_{e}}{d x} \\
+v \frac{3(n-1) \Gamma^{2}}{2}\left(\frac{\partial u}{\partial y}\right)^{2} \frac{\partial^{2} u}{\partial y^{2}} \\
+\frac{\mu}{4 \pi \rho}\left(H_{1} \frac{\partial H_{1}}{\partial x}+H_{1} \frac{\partial H_{2}}{\partial y}\right) \\
u \frac{\partial H_{1}}{\partial x}+v \frac{\partial H_{1}}{\partial y}=\mu_{e} \frac{\partial^{2} H_{1}}{\partial y^{2}}+H_{1} \frac{\partial u}{\partial x}+H_{2} \frac{\partial u}{\partial y} \\
u \frac{\partial T}{\partial x}+v \frac{\partial T}{\partial y}=\frac{k_{f}}{\rho C_{p}} \frac{\partial^{2} T}{\partial y^{2}}
\end{gathered}
$$

where $u$ and $v$ are the velocity and $H_{1}$ and $H_{2}$ are the magnetic components along the $x-$ and $y-$ axes respectively, $v$ is the kinematic viscosity, $\rho$ is the density of the fluid, $\mu$ is the magnetic permeability, $\mu_{e}$ is the magnetic diffusivity, $T$ is the temperature of the fluid, $k_{f}$ is the thermal conductivities of the fluid and $\rho C_{p}$ is the heat capacity of the fluid.

The boundary conditions of these equations are

$$
\begin{gathered}
u=u_{w}(x)=a x, \quad v=v_{w}(x)=b x, \quad \frac{\partial H_{1}}{\partial y}=0, \\
H_{2}=0, \quad k_{f} \frac{\partial T}{\partial y}=-h_{f}\left(T_{f}-T\right) \quad \text { at } \quad y=0, \\
u=u_{e} \rightarrow b x, \quad H_{1}=H_{e}(x) \rightarrow H_{0}(x), \\
T \rightarrow T_{\infty} \quad \text { as } \quad y \rightarrow \infty
\end{gathered}
$$

where $a$ and $b$ are constants, $T_{f}$ is the temperature of convective fluid, $h_{f}$ is the convective heat transfer coefficient, $H_{e}(x)$ is the $x$ component of magnetic field at the edge of the boundary layer and $H_{0}(x)$ is the applied magnetic field parallel to the $x$ - axis in the free stream. In order to transform Eqs. (3)-(8) into a set of ordinary differential equations, the following similarity variable are introduced

$$
\begin{gathered}
u=b x f^{\prime}(\eta), v=-(b v)^{1 / 2} f(\eta), \\
H_{1}=H_{0} x h^{\prime}(\eta), \quad H_{2}=-\left(\frac{v}{b}\right)^{1 / 2} H_{0} h(\eta), \\
\theta(\eta)=\frac{T-T_{\infty}}{T_{w}-T_{\infty}}, \quad \eta=\left(\frac{b}{v}\right)^{1 / 2} y
\end{gathered}
$$

where primes denote differentiation with respect to $\eta$.

Subtituting (9) into Eqs. (3)-(8), we obtain the following ordinary differential equations 


$$
\begin{gathered}
f^{\prime \prime \prime}+f f^{\prime \prime}-f^{\prime 2}+1+\frac{3(n-1) W e^{2}}{2} f^{\prime \prime \prime}\left(f^{\prime \prime}\right)^{2} \\
+M\left(h^{\prime 2}-h h^{\prime \prime}-1\right)=0 \\
\chi h^{\prime \prime \prime}+f h^{\prime \prime}-f^{\prime \prime} h=0 \\
\frac{1}{P r} \theta^{\prime \prime}+f \theta^{\prime}=0
\end{gathered}
$$

subjected to the boundary conditions

$$
\begin{gathered}
f(0)=S, f^{\prime}(0)=\lambda, h(0)=0, h^{\prime \prime}(0)=0, \\
\theta^{\prime}(0)=-B i[1-\theta(0)], \\
f^{\prime}(\infty) \rightarrow 1, \quad h^{\prime}(\infty) \rightarrow 1, \quad \theta(\infty) \rightarrow 0 .
\end{gathered}
$$

In the above equations, $W e^{2}=\frac{b^{3} x^{2} \Gamma^{2}}{v}$ is the Weissenberg number, $\quad M=\frac{\mu H_{0}^{2}}{4 \pi \rho b^{2}} \quad$ is the magnetic parameter, $\chi=\frac{\mu_{e}}{v}$ is the reciprocal magnetic parameter, $\operatorname{Pr}=\frac{v}{\alpha}$ is the Prandtl number, $S=-\frac{v_{w}}{(b v)^{1 / 2}}$ is the suction/injection parameter, $\quad \lambda=\frac{a}{b} \quad$ is the stretching/shrinking parameter and $B i=\frac{h_{f}}{k_{f}}\left(\frac{v}{b}\right)^{1 / 2}$ is the Biot number or convective parameter.

The physical quantities of interest are the skin coefficient $C_{f}$ and the local Nusselt number $N u_{x}$, which defined as

$$
C_{f}=\frac{\tau_{w}}{\rho_{f} u_{w}^{2}}, \quad N u_{x}=\frac{x q_{w}}{k_{f}\left(T_{w}-T_{\infty}\right)}
$$

where $\tau_{w}$ is the skin friction or shear stress and $q_{w}$ is the surface heat flux given by

$$
\begin{gathered}
\tau_{w}=\frac{\partial u}{\partial x}+\frac{(n-1) \Gamma^{2}}{2}\left(\frac{\partial u}{\partial y}\right)_{y=0}^{3}, \\
q_{w}=-k_{f}\left(\frac{\partial T}{\partial y}\right)_{y=0} .
\end{gathered}
$$

Using the similarity variables (9), we obtain

$$
C_{f} \operatorname{Re}_{x}^{1 / 2}=f^{\prime \prime}(0), \frac{N u_{x}}{R e_{x}^{1 / 2}}=-\theta^{\prime}(0)
$$

where $R e_{x}=\frac{u_{e} x}{v}$ is the local Reynolds number.

\section{STABILITY ANALYSIS}

To conduct the stability analysis, we consider the problem as unsteady case. The continuity Eqs. (3) and (4) hold, while Eqs. (5)-(7) are replaced by

$$
\begin{array}{r}
\frac{\partial u}{\partial t}+u \frac{\partial u}{\partial x}+v \frac{\partial u}{\partial y}=v \frac{\partial^{2} u}{\partial y^{2}}+u_{e} \frac{d u_{e}}{d x} \\
+v \frac{3(n-1) \Gamma^{2}}{2}\left(\frac{\partial u}{\partial y}\right)^{2} \frac{\partial^{2} u}{\partial y^{2}} \\
+\frac{\mu}{4 \pi \rho}\left(H_{1} \frac{\partial H_{1}}{\partial x}+H_{1} \frac{\partial H_{2}}{\partial y}\right)-\frac{\mu H_{e}}{4 \pi \rho} \frac{d H_{e}}{d x} \\
\frac{\partial H_{1}}{\partial t}+u \frac{\partial H_{1}}{\partial x}+v \frac{\partial H_{1}}{\partial y}=\mu_{e} \frac{\partial^{2} H_{1}}{\partial y^{2}} \\
+H_{1} \frac{\partial u}{\partial x}+H_{2} \frac{\partial u}{\partial y} \\
\frac{\partial T}{\partial t}+u \frac{\partial T}{\partial x}+v \frac{\partial T}{\partial y}=\frac{k}{\rho C_{p}} \frac{\partial^{2} T}{\partial y^{2}}
\end{array}
$$

where $t$ denotes the time. We now introduce the following new dimensionless variables:

$$
\begin{gathered}
\eta=\left(\frac{b}{v}\right)^{1 / 2} y, \quad \tau=a t, \quad u=b x f^{\prime \prime}(\eta, \tau), \\
v=-(b v)^{1 / 2} f(\eta, \tau), \quad H_{1}=H_{0} x h^{\prime}(\eta, \tau), \\
H_{2}=-\left(\frac{v}{b}\right)^{1 / 2} H_{0} h(\eta, \tau), \theta(\eta, \tau)=\frac{T-T_{\infty}}{T_{w}-T_{\infty}}
\end{gathered}
$$

so that Eqs. (17)-(19) can be written as

$$
\begin{gathered}
\frac{\partial^{3} f}{\partial \eta^{3}}-\left(\frac{\partial f}{\partial \eta}\right)^{2}+f \frac{\partial^{2} f}{\partial \eta^{2}}+1 \\
+\frac{3(n-1) W e^{2}}{2}\left(\frac{\partial^{2} f}{\partial \eta^{2}}\right)\left(\frac{\partial^{2} f}{\partial \eta^{3}}\right)^{2} \\
+M\left[\left(\frac{\partial h}{\partial \eta}\right)^{2}-h\left(\frac{\partial^{2} h}{\partial \eta^{2}}\right)-1\right]-\frac{\partial^{2} f}{\partial \eta \partial \tau}=0 \\
\chi \frac{\partial^{3} h}{\partial \eta^{3}}+f \frac{\partial^{2} h}{\partial \eta^{2}}-h \frac{\partial^{2} f}{\partial \eta^{2}}-\frac{\partial^{2} h}{\partial \eta \partial \tau}=0 \\
\frac{1}{\operatorname{Pr}} \frac{\partial^{2} \theta}{\partial \eta^{2}}+f \frac{\partial \theta}{\partial \eta}-\frac{\partial \theta}{\partial \tau}=0
\end{gathered}
$$

and are subjected to the boundary conditions 


$$
\begin{gathered}
f(0, \tau)=S, \quad \frac{\partial f}{\partial \eta}(0, \tau)=\lambda, \quad h(0, \tau)=0, \\
\frac{\partial^{2} h}{\partial \eta^{2}}(0, \tau)=0, \quad \frac{\partial \theta}{\partial \eta}(0, \tau)=-B i[1-\theta(0, \tau)], \\
\frac{\partial f}{\partial \eta}(\eta, \tau) \rightarrow 1, \quad \frac{\partial h}{\partial \eta}(\eta, \tau) \rightarrow 0 \\
\theta(\eta, \tau) \rightarrow 0 \quad \text { as } \quad \eta \rightarrow \infty .
\end{gathered}
$$

To test the stability of the steady flow solution, we use $f(\eta)=f_{0}(\eta), h(\eta)=h_{0}(\eta)$ and $\theta(\eta)=\theta_{0}(\eta)$ which satisfying the boundary value problem (10)-(13). Hence, we write

$$
\begin{aligned}
& f(\eta, \tau)=f_{0}(\eta)+e^{-\gamma \tau} F(\eta, \tau), \\
& h(\eta, \tau)=h_{0}(\eta)+e^{-\gamma \tau} H(\eta, \tau), \\
& \theta(\eta, \tau)=\theta_{0}(\eta)+e^{-\gamma \tau} T(\eta, \tau),
\end{aligned}
$$

where $\gamma$ is an unknown eigenvalue, $F(\eta, \tau), H(\eta, \tau)$ and $T(\eta, \tau)$ are small relative to $f_{0}(\eta), h_{0}(\eta)$ and $\theta_{0}(\eta)$. Solutions of the eigenvalue problem in Eqs. (21)-(24) give an infinite set of eigenvalue problem $\gamma_{1}<\gamma_{2}<\ldots$; if $\gamma_{1}$ is negative, there is an initial growth of disturbances and the flow is unstable but when $\gamma_{1}$ is positive, there is an initial decay and the flow is stable. Introducing (25) into Eqs. (21)(24), we get the following linearized problem

$$
\begin{gathered}
\frac{\partial^{3} F}{\partial \eta^{3}}+f_{0} \frac{\partial^{2} F}{\partial \eta^{2}}+f_{0}^{\prime \prime} F-\left(2 f_{0}^{\prime}-\gamma\right) \frac{\partial F}{\partial \eta} \\
+\frac{3(n-1) W e^{2}}{2}\left[2 f_{0}^{\prime \prime \prime} f_{0}^{\prime \prime} \frac{\partial^{2} F}{\partial \eta^{2}}+\left(f_{0}^{\prime \prime}\right)^{2} \frac{\partial^{3} F}{\partial \eta^{3}}\right] \\
+M\left[2 h_{0}^{\prime} \frac{\partial H}{\partial \eta}-h_{0} \frac{\partial^{2} H}{\partial \eta^{2}}-h_{0}^{\prime \prime} H\right]-\frac{\partial^{2} F}{\partial \eta \partial \tau}=0 \\
\chi H_{0}^{\prime \prime \prime}+f_{0} H_{0}^{\prime \prime}+h_{0}^{\prime \prime} F_{0}-f_{0}^{\prime \prime} H_{0}-h_{0} F_{0}^{\prime \prime} \\
+\gamma H_{0}^{\prime}=0
\end{gathered}
$$

$$
\frac{1}{P r} T_{0}^{\prime \prime}+f_{0} T_{0}^{\prime}+\theta_{0}^{\prime} F_{0}+\gamma T_{0}=0
$$

The stability of the steady-state flow solution is based on the smallest eigenvalue $\gamma_{1}$. Therefore, the condition $F_{0}^{\prime}(\eta) \rightarrow 0$ has been put at rest as suggested by Harris et al.(2009) and for a fixed value of eigenvalue $\gamma$. Eqs. (30)(33) will be solved by introducing a new boundary condition that is $F_{0}^{\prime \prime}(0)=1$.

\section{RESULTS AND DISCUSSIONS}

Equations (10)-(12) subject to the boundary conditions (13) have been solved numerically using the bvp4c function in Matlab solver. To validate the accuracy of this present study, the numerical result for the skin friction coefficient when the magnetic parameter is neglected $(M=0)$ are compared with the previous published studies. The data obtained shows a good agreement with Bhattacharyya (2011) and Akbar et. al., (2014) as presented in Table 1. Figure 1 displays the effect of suction parameter on velocity profile. It is observed that for increasing value of suction parameter $S$, the boundary layer thickness is decreasing for upper and lower solutions in the profile. The effect of reciprocal magnetic parameter $\chi$ on induced magnetic profile can be seen from figure 2. It seen clearly that the boundary layer thickness increases as the value of reciprocal magnetic parameter $\chi$ increases.

Table 1. Comparison values of the skin friction coefficient $f^{\prime \prime}(0)$ for some values of $\lambda$ when $M=0$

\begin{tabular}{ccccccc}
\hline & \multicolumn{2}{c}{ Bhattacharyya (2011) } & \multicolumn{2}{c}{ Akhbar et al. (2014) } & \multicolumn{2}{c}{ Present } \\
\cline { 2 - 6 }$\lambda$ & First & Second & First & Second & First & Second \\
& Solution & Solution & Solution & Solution & Solution & Solution \\
\hline-0.25 & 1.4022 & - & 1.4022 & - & 1.40224 & - \\
-0.50 & 1.4957 & - & 1.4957 & - & 1.49567 & - \\
-0.75 & 1.4893 & - & 1.4893 & - & 1.48929 & - \\
-1.00 & 1.3288 & 0 & 1.3288 & 0 & 1.32882 & 0 \\
-1.15 & 1.0822 & 0.1167 & 1.0822 & 0.1167 & 1.08223 & 0.11670 \\
-1.20 & 0.9325 & 0.2336 & 0.9325 & 0.2336 & 0.93247 & 0.23365 \\
\hline
\end{tabular}




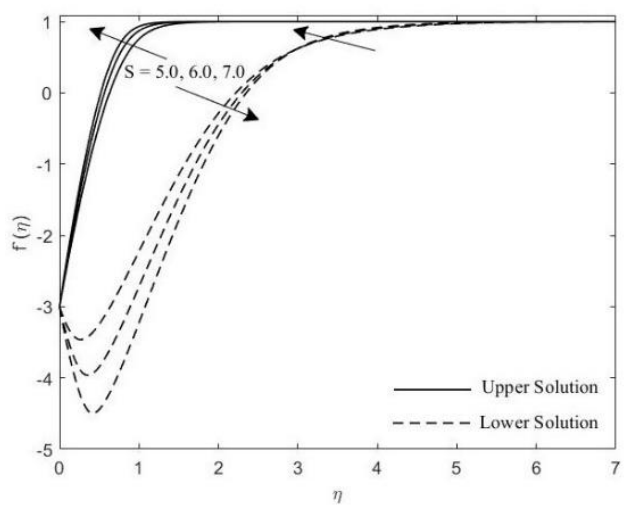

Figure 1. Velocity profile for various value of $S$

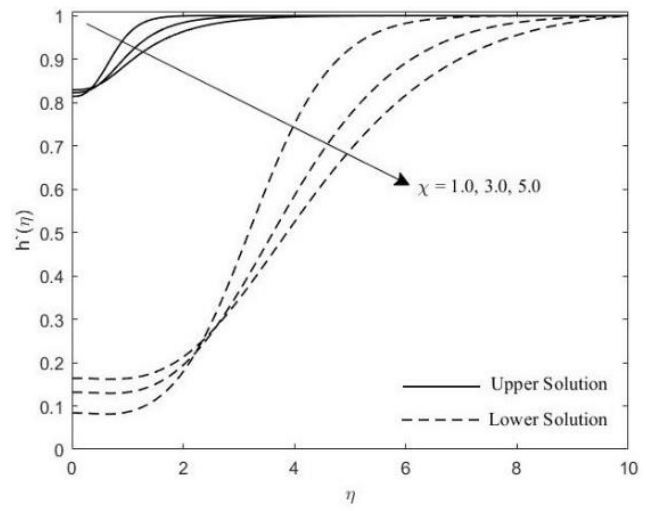

Figure 2. Induced magnetic profile for various value of $\chi$

Next, figure 3 shows the effect of Prandtl number $\mathrm{Pr}$ on temperature profile. It noticed that as the value of $\mathrm{Pr}$ increases, the thermal boundary layer thickness decreases. On the other hand, it is seen from figure 4 for increasing values of Biot number $B i$ it increases the thermal boundary layer thickness on temperature profile. When the Biot number increases, the convection becomes stronger, hence it increases the surface temperature. Therefore, it is causing the thermal effect to penetrate deeper into fluid. Figures 510 illustrate the dual solutions for the physical quantities of interest, which are the skin friction coefficient $f^{\prime \prime}(0)$ and the local Nusselt number $-\theta^{\prime}(0)$. Based on the observation, dual solutions of Eqs. (10)-(12) can be obtained for both stretching and shrinking cases.

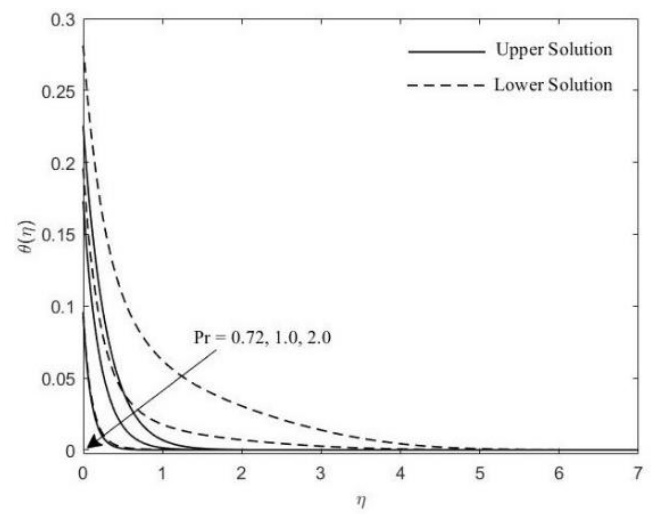

Figure 3. Temperature profile for various value of $\mathrm{Pr}$

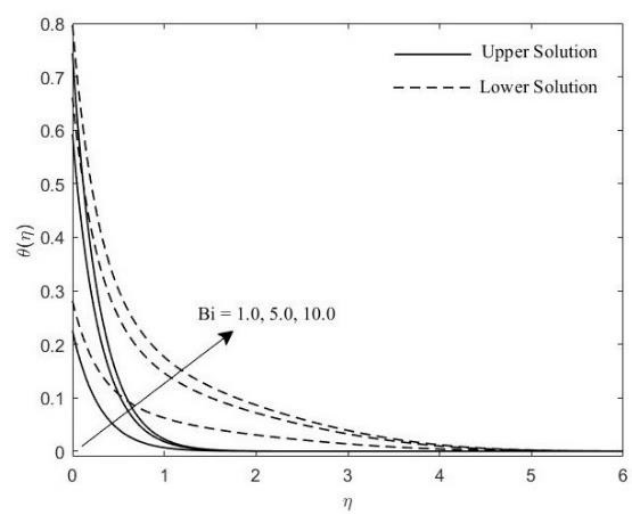

Figure 4. Temperature profile for various value of $B i$

From figures 5 and 6 , we can see that as the values of magnetic parameter $M$ increase, the skin friction coefficient $f^{\prime \prime}(0)$ and local Nusselt number $-\theta^{\prime}(0)$ decreases. This is due to the fact that the presence of transverse magnetic field sets in Lorentz force, which results in retarding force on the velocity field. This force has the tendency to slow down the motion of the fluid in the boundary layer. As a result, the boundary layer separation occurs quicker when the magnetic field is applied. Therefore, the magnetic field enhances the boundary layer separation. The effects of power law index $n$ and Weissenberg number $W e$ on the skin friction coefficient $f^{\prime \prime}(0)$ and local Nusselt number $-\theta^{\prime}(0)$ are shown in figures 7-10, respectively. Based on the critical value $\lambda_{c}$ in these figures, the boundary layer separation occurs faster as power law index $n$ and Weissenberg number increase. 


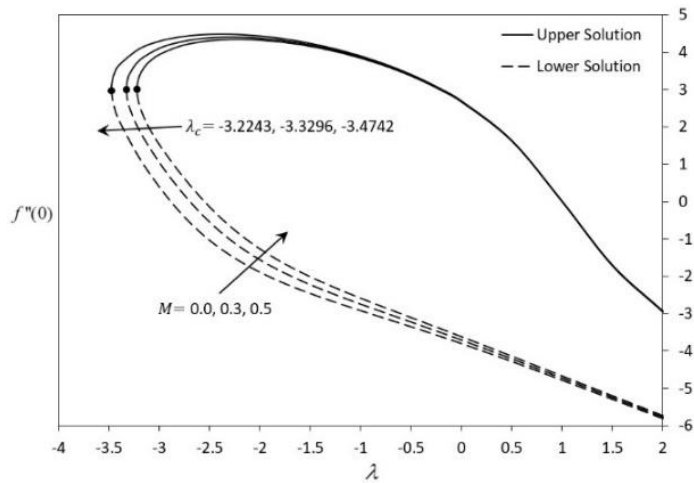

Figure 5. Variation of skin friction coefficient for various $M$

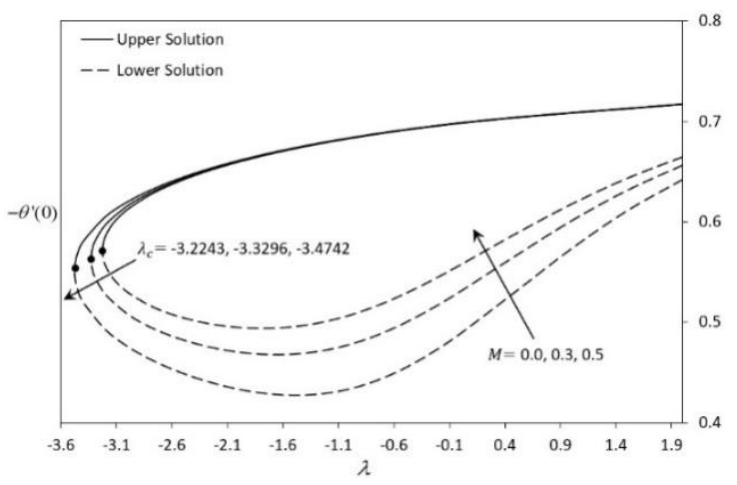

Figure 6. Variation of local Nusselt number for various M

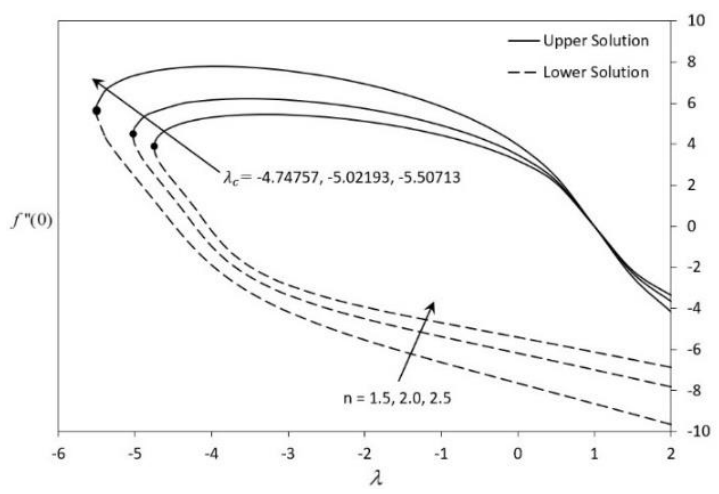

Figure 7. Variation of skin friction coefficient for various $n$

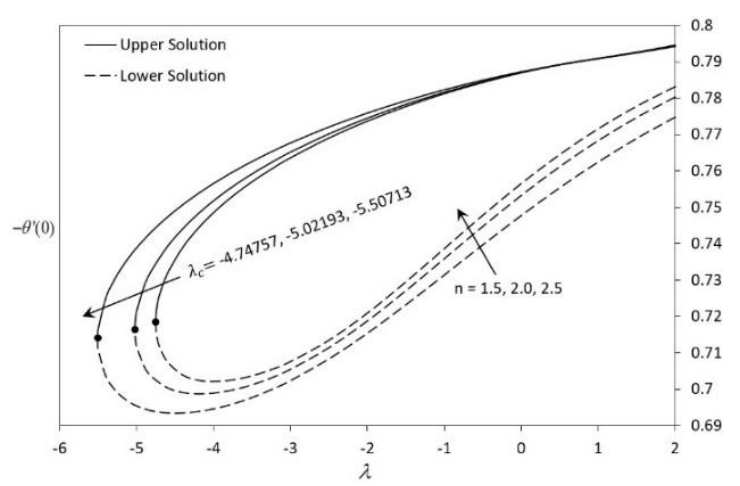

Figure 8. Variation of local Nusselt number for various $n$

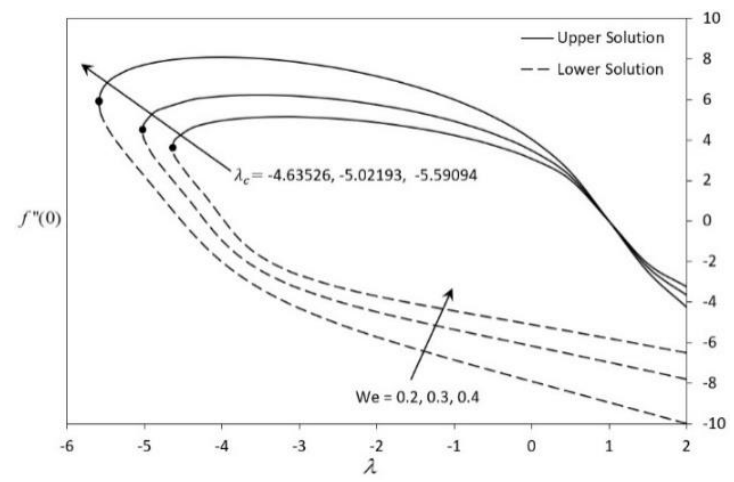

Figure 9. Variation of skin friction coefficient for various $W e$

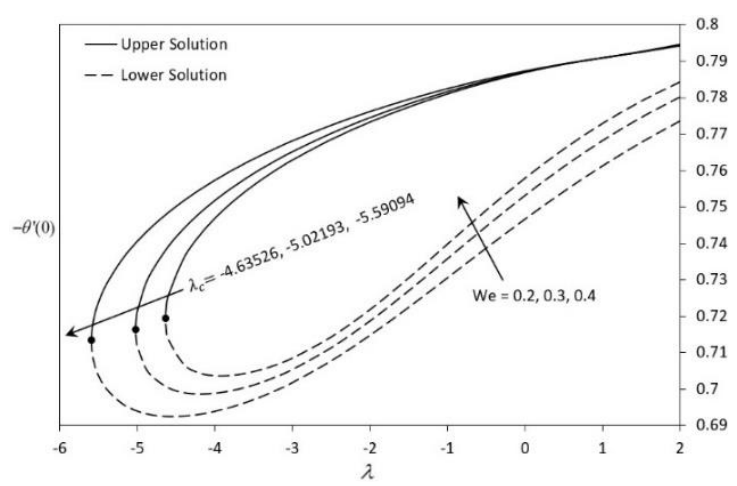

Figure 10. Variation of local Nusselt number for various $W e$

Based on all these figures, it is clearly shown that the existence of non-unique solution. Therefore, it is our determination to show whether the first solution is stable and realizable physically or otherwise. Therefore, the eigenvalue problem (30)-(33) is solved for the smallest eigenvalues $\gamma_{1}$ on the upper and lower solution branches. These results are presented in Table 2 for several values of $\lambda$ when $M=0.1,0.3,0.5$. 
Table 2: Smallest eigenvalue $\gamma_{1}$ for some values of $\lambda$ with different $M$

\begin{tabular}{cccc}
\hline$M$ & $\lambda$ & First solution & Second solution \\
\hline \multirow{3}{*}{0.1} & 3.4 & 0.69576 & -0.60028 \\
& 3.42 & 0.37946 & -0.29936 \\
& 3.427 & 0.12595 & -0.05125 \\
\hline \multirow{2}{*}{0.3} & 3.3 & 0.60902 & -0.60639 \\
& 3.32 & 0.34824 & -0.34654 \\
& 3.329 & 0.08742 & -0.08613 \\
0.5 & 3.2 & 0.45343 & -0.52959 \\
& 3.22 & 0.17612 & -0.24112 \\
& 3.224 & 0.02718 & -0.08991 \\
\hline
\end{tabular}

From the table, it is observed that the smallest eigenvalue $\gamma_{1}$ gives a positive value for the first solution and negative value for the second solution. Hence, we can draw a conclusion that only the upper branch solutions are physically significant while the lower branch solutions are not. Furthermore, as the value of $\lambda$ approaching the critical point $\lambda_{c}$, the smallest eigenvalue $\gamma_{1}$ converges to 0 for both upper and lower branches as discovered by Merkin (1985).

\section{CONCLUSION}

A numerical study is performed for the problem of the steady two-dimensional magnetohydrodynamics (MHD) stagnation-point flow of Carreau fluid towards a permeable stretching/shrinking sheet in the presence of induced magnetic field. The convective boundary condition also taken into account. The effect of all parameters is investigated numerically and graphically. Dual solutions found to exist for both stretching and shrinking cases. Therefore, stability analysis is done via bvp4c function in MATLAB software and their results found that the first solution (upper branch) is stable and valid physically, while the second solution (lower branch) is not stable.

\section{ACKNOWLEDGEMENT}

The authors gratefully acknowledge the financial received in the form Geran Putra IPS (Project number: GPIPS/2018/9570000) from the Universiti Putra Malaysia and $\mathrm{MyPhD}$ from the Ministry of Higher Education, Malaysia. 


\section{REFERENCES}

Adnan, N. S. M. \&Arifin, N. M. 2017, Stability analysis of boundary layer flow and heat transfer over a permeable exponentially shrinking sheet in the presence of thermal radiation and partial slip. Journal of Physics: Conference Series, 890,012046.

Akbar, N. S., Nadeem, S., Haq, R. U. \& Ye, S. 2014, MHD stagnation point flow of Carreau fluid toward a permeable shrinking sheet: Dual solutions. Ain Shams Engineering Journal, 5(4), pp. 1233-1239.

Bhattacharyya, K. 2011, Dual solutions in unsteady stagnation-point flow over a shrinking sheet. Chinese Physics Letters, 28(8), 084702.

Carreau, P. J. 1972, Rheological Equations from Molecular Network Theories. Transactions of the Society of Rheology, 16, pp. 99-127.

Harris, S. D., Ingham, D. B., \& Pop, I. 2009, Mixed convection boundary-layer flow near the stagnation point on a vertical surface in a porous medium: Brinkman model with slip. Transport in Porous Media, 77, pp. 267-285.

Hayat, T., Asad, S., Mustafa, M. \& Alsaedi, A. 2014 Boundary layer flow of Carreau fluid over a convectively heated stretching sheet. Applied Mathematics and Computation, 246, pp. 12-22.

Junoh, M. M., Ali, F. M., Arifin, N. M. \& Bachok, N. 2018, Dual solutions in magnetohydrodynamic (MHD) flow on a nonlinear porous shrinking sheet: A stability analysis. AIP Conference Proceedings, 1974, 020083.

Khan, M. \&Hashim. 2015, Boundary layer flow and heat transfer to Carreau fluid over a nonlinear stretching sheet. AIP Advances, 5(10), 107203.

Khan, M., Hashim \& Alshomrani, A.S. 2016, MHD stagnation-point flow of a Carreau fluid and heat transfer in the presence of convective boundary conditions. PLoS ONE, 11(6), e0157180.

Khan, M., Sardar, H., Gulzar, M. M., \& Alshomrani, A. S. 2018, On multiple solutions of non-Newtonian Carreau fluid flow over an inclined shrinking sheet. Results in Physics, 8, pp. 926-932.
Khellaf, K. \& Lauriat, G. 2000, Numerical study of heat transfer in a non-Newtonian Carreau fluid between rotating concentric vertical cylinders. Journal of NonNewtownian Fluid Mechanics, 89(1), pp. 45-61.

Malik, M. Y., Zehra, I. \&Nadeem, S. 2014, Flows of Carreau fluid with pressure dependent viscosity in variable porous medium: Application of polymer melt. Alexandria Engineering Journal, 53(2), pp. 427-435.

Merkin, J. H. 1985, On dual solutions occuring in mixed convection in a porous medium. Journal of Engineering Mathematics, 20, pp. 171-179.

Suneetha, S. \&Gangadhar, K. 2015, Thermal radiation effect on MHD stagnation point flow of a Carreau fluid with convective boundary condition. Open Science Journal of Mathematics and Application, 3(5), pp. 121-127.

Weidman, P. D., Kubitschek, D. G., \& Davis, A. M. J. 2006, The effect of transpiration on self-similar boundary layer flow over moving surfaces. International Journal of Engineering Sciences, 44, pp. 730-737. 